\title{
Fine-scale urban heat patterns in New York City measured by ASTER satellite - the role of complex spatial structures
}

Bibhash Nath $^{\mathrm{a}^{*} \text {, Mutlu Özdoğan }}{ }^{\mathrm{b}}$,Wenge Ni-Meister ${ }^{\mathrm{a}}$

${ }^{\text {a }}$ Department of Geography and Environmental Science, Hunter College of the City University of New York, NY 10021, USA

${ }^{\mathrm{b}}$ Department of Forest and Wildlife Ecology, University of Wisconsin-Madison, Madison, WI 53706, USA

*Corresponding author: Bibhash Nath, e-mail: bibhash12@gmail.com and bibhash.nath86@myhunter.cuny.edu 


\begin{abstract}
Urban areas have very complex spatial structures. These spatial structures are primarily composed of a complex network of built environments, which evolve rapidly as the cities expand to meet the growing population's demand and economic development. Therefore, studying the impact of spatial structures on urban heat patterns is extremely important for sustainable urban planning and growth. We investigated the relationship between surface temperature obtained by the Advanced Spaceborne Thermal Emission and Reflection Radiometer (ASTER, at $90 \mathrm{~m}$ spatial resolution) on the current EOS-Terra platform and different urban components based on the classification of high-resolution QuickBird imagery. We further investigated the relationships between surface temperature and building footprint and land use information acquired from the New York City (NYC) Department of City Planning. The ASTER image reveals fine-scale urban heat patterns in the NYC metropolitan region. The dark and medium-dark impervious surfaces, along with bright surfaces, generate higher surface temperatures. Even with highly reflective urban materials, the presence of impervious materials leads to an increased surface temperature. At the same time, trees and shadows are effective in reducing urban heat. The data aggregated to the census tract reveals high-temperature clusters in Queens, Brooklyn, and the Bronx region of NYC. These clusters are associated with industrial and manufacturing areas and multi-family walk-up buildings as dominant land use. The census tracts with more trees and higher building height variability generate lower surface temperatures, consistent with shadow cast by high-rise buildings and trees. The results of this study can be valuable for urban heat island modeling on the effects of building heights variability and tree shadows on small-scale surface temperature patterns. It can also help identify the risk areas during extreme heat events to protect public health.
\end{abstract}

Keywords: Urban Heat Island, Building Footprint, Remote Sensing, ASTER, New York City. 


\section{Introduction}

Urban areas have very complex spatial structures (Zhou et al., 2017). These spatial structures continue to evolve rapidly as the cities expand to meet the growing population's demand and economic development. The intensity of these transformations during recent times is at an alarming level worldwide. Because, at present, 55\% of the world's population (4.2 billion) resides in urban areas, and the percentage is expected to rise to $70 \%$ by 2050 (Callaghan et al., 2021). The transformations of urban areas result in higher surface- and air- temperatures than rural areas, which is termed as the "urban heat island (UHI) effect" (Oke, 1982; Tan et al., 2019). The UHI effect leads to environmental changes, climate, vegetation growth, air and water quality (Zhou et al., 2019) and threatens human health and well-being. Therefore, understanding UHI and its causes are crucial to managing urban expansion and mitigating UHI effects.

The UHI is often classified into three categories. The first category is the boundary layer UHI, which occurs in the layer above the mean building height. The second category is the canopy layer UHI, which occurs in the layer from the surface to approximately the mean building height. The third category is the surface UHI (SUHI), which occurs at the surface (Voogt and Oke, 2003). The first two categories are studied based on ground-based meteorological measurements or intensive field experiments within a limited period of time (Voogt and Oke, 2003). Lack of spatial samplings of surface temperatures in and around cities has been recognized as a significant problem in the traditional atmosphere UHI study (Voogt and Oke, 1997). On the other hand, the SUHI refers to the relative temperature differences of urban surfaces compared to surrounding rural areas. Our study is focused on SUHI.

Factors causing SUHI can be categorized into three categories: physical, structural, and anthropogenic factors. Heat-trapping and non-porous urban surface materials absorb more heat 
and prevent heat release back to the atmosphere. The UHI can be complicated by the land-sea breeze's cooling effect (Yoshikado, 1990, 1992). Complex urban geometry casts shadows between high-rise buildings, reduces sun exposure, and narrows the surface view of the atmosphere, resulting in a lower surface temperature (Nichol and Wong, 2005; Guo et al., 2016). Anthropogenic factors, such as winter heating or summer cooling systems from commercial and residential buildings, amplify the intensity of the UHIs. Trees in the neighborhood increase the likelihood of cooler surface temperature because of excess heat released by trees through evapotranspiration. The SUHI is typically characterized by airborne or satellite thermal infrared remote sensing at regional scales. The recent developments in spaceborne and airborne remote sensing technology and the availability of thermal sensing data from various satellites makes it possible to study SUHI effectively (Voogt and Oke, 2003; Zhou et al., 2019). Our study focuses on using thermal remote sensing data to examine the spatial structure of urban thermal patterns and their relation to urban surface characteristics.

In big cities, tall buildings often cast shadows on buildings and the ground. Loughner et al. (2012) observed that higher surface temperature is associated with shorter urban buildings based on the model simulation. They concluded that shorter buildings cast fewer shadows and allow heating of the building walls and roads through direct sunlight (Loughner et al., 2012). On the other hand, taller buildings cast larger shadows and reduces solar radiation absorption onto urban impervious surfaces. The availability of high-resolution imagery from satellite sensors such as IKONOS and QuickBird provides a unique opportunity for detailed urban land cover mapping. We can obtain accurate urban land use information, including impervious surfaces, vegetation cover, spatial organization of urban buildings, shadows, and surface temperature patterns. 
The pixel-based thermal signal over urban areas is represented as a mixture of different urban components, resulting in irresolvable hot and cold spots (Price 1979; Carlson 1986). Roth et al. (1989) observed the effect of a few very hot roofs or a significant combustion source heat on the pixel values in an area where most of the active surface is much cooler. The remotely sensed surface temperature exhibits large spatial variability (i.e., the mixture of cold and hot surface components) together with a small spatial scale of urban structure (typically between $10 \mathrm{~m}$ and 20 m) (Roth et al., 1989; Voogt and Oke, 2003). Therefore, it is necessary to unmix the satellite observed mixed temperature to derive surface temperature for different urban components to better use satellite observed UHI (Voogt and Grimmond, 2000). In addition, the availability of urban structural information provides a unique opportunity to combine field-based building footprint information with remote sensing measurements to explain urban heat patterns at a finer scale.

This study investigates the factors causing fine-scale spatial variations in urban heat patterns in the New York City (NYC) metropolitan region. We attempted to establish a relation between surface temperature measured by ASTER and urban structures, urban materials, shadows, and vegetation cover, identified by classification of very high resolution $(2.8 \mathrm{~m})$ QuickBird imagery and the urban structural data (i.e., building footprint information and dominant land use category) obtained from the NYC Department of City Planning. We further examined the role of urban trees and building height on the SUHI in the NYC metropolitan region during autumn when the Sun is at a lower elevation and the likelihood of producing more shadows by tall urban structures.

\section{Study area}

The study area is located in New York City (NYC) and its metropolitan area (Fig. 1). A true-color composite of ASTER satellite (15 m spatial resolution) shows the fine detail of urban structures: 
vegetation in green, urban areas in white/brown, and water in black (Fig. 1). The residential areas show scattered bright spots mixed with green trees on the streets. At the center is Manhattan, with a bright green oasis (Central Park) in the middle. Manhattan is the center of the financial district with many tall buildings mainly located south of Central Park in the downtown and midtown region. The dark color represents the shadows cast by the high-rise buildings. The mixed bright and dark spots in the west part of the midtown are lower-rise buildings. The Hudson River runs north to south and separates New Jersey (on the left) from Manhattan Island (on the right). The Harlem River at the north of Manhattan separates Manhattan from the Bronx. The Harlem River merges with the East River and separates Manhattan from Queens in the east and Brooklyn in the southeast. Following the direction to which Manhattan points is the Staten Island. In the suburban region, green vegetation is scattered in the residential backyards. The industrial areas shown in bright and dark spots are located at the intersection between Queens and Brooklyn, next to the East River (Fig. 1b). 


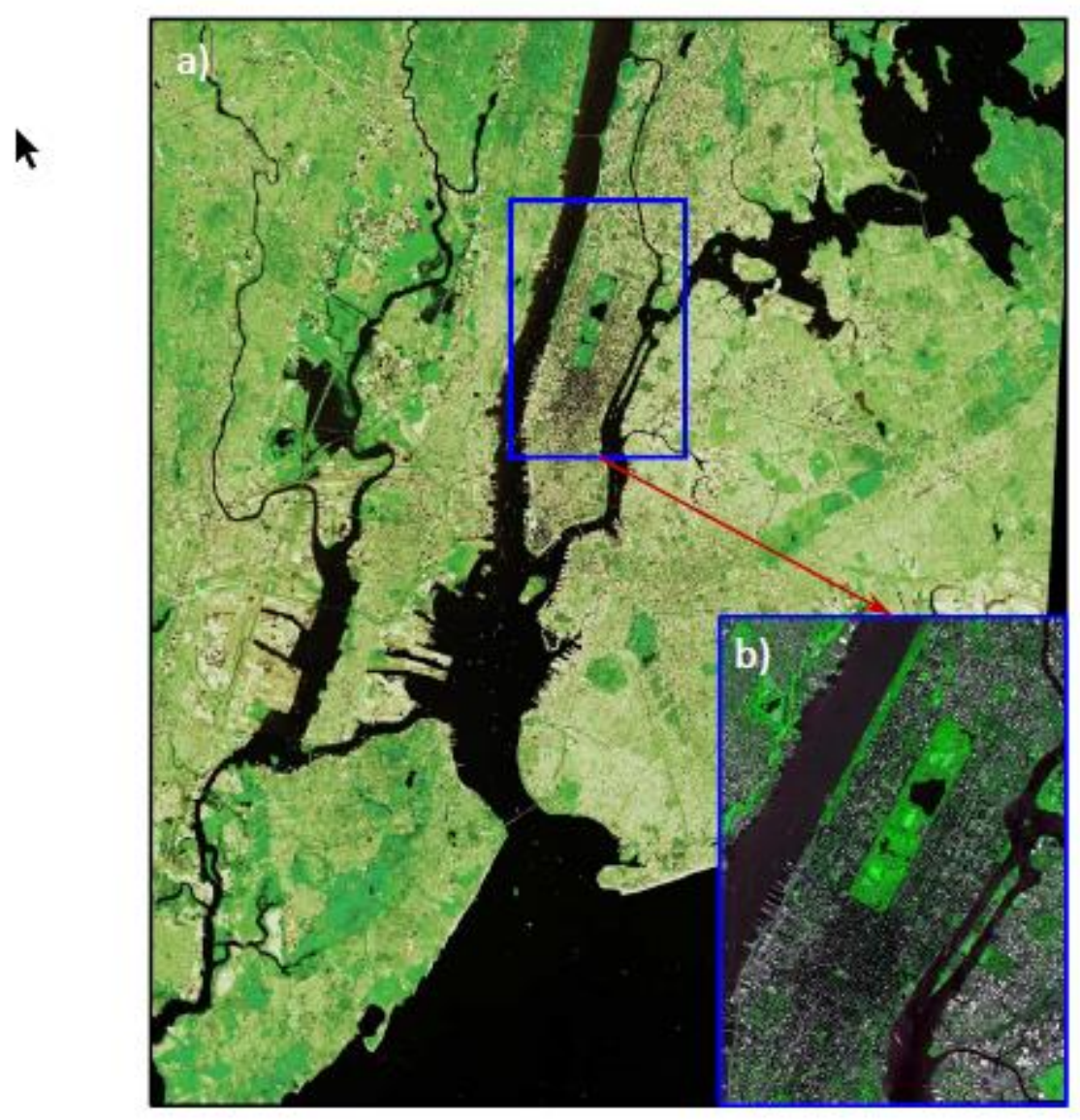

Figure 1. Overview of the study area: a) True color EOS-Terra ASTER satellite image of the New York City Metropolitan area observed on September 8, 2002, and b) True color QuickBird satellite in Manhattan and its surrounding area (blue square) observed on August 2, 2002.

The climate is controlled by cold, dry air mass movement from the north and warm, humid air mass movement from the south. In addition to the above two air masses' interactions, the air mass flow from the North Atlantic Ocean produces cool, cloudy, and damp weather conditions in the NYC metropolitan area. The land-sea temperature differences in all seasons have a strong local impact on NYC climate. Land-sea breezes cool NYC on warm spring and summer days and warm NYC on cold nights in autumn, winter, and early spring (Gedzelman et al., 2003). Surface elevation increases from the coast to inland. In the NYC metropolitan region, two ridges are parallel to the 
coastline (Fig. 2a). One is along the boundary between Brooklyn and Queens, and the other is on the west side of the Hudson River. Each ridge blocks the cooler land-sea breeze when moving from the coast to inland, resulting in warmer surface temperatures (Fig. 2b).
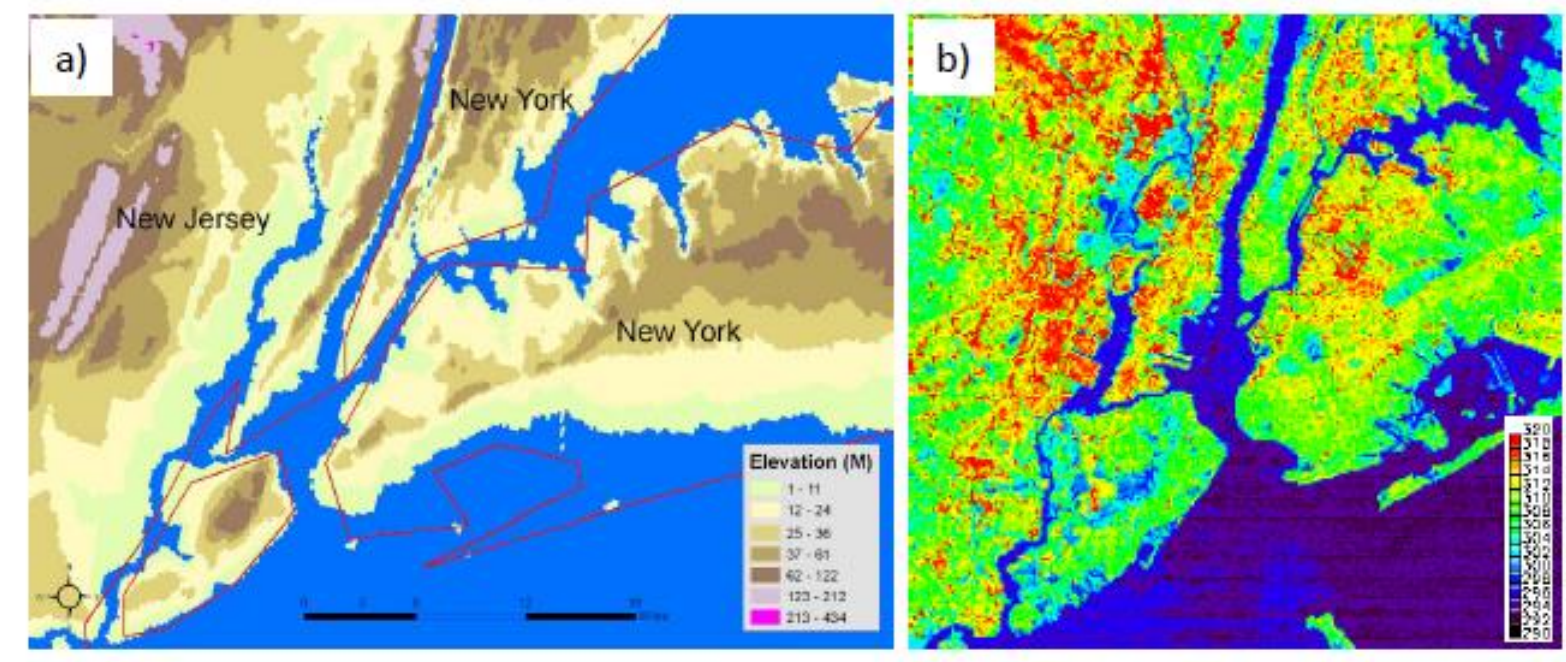

Figure 2. The maps showing: a) The topography of New York City (at $80 \mathrm{~m}$ spatial resolution), two ridges can be seen in the map, and b) ASTER surface temperature (K) over New York City and surrounding locations on September 8, 2002.

\section{Materials and methods}

\subsection{Data source}

Advanced Spaceborne Thermal Emission and Reflection Radiometer (ASTER) level 2 Surface Kinetic Temperature (AST_08, at $90 \mathrm{~m}$ spatial resolution) with an accuracy of 1.5 Kelvin was downloaded from NASA (ASTER_08, 2020, Yamaguchi et al., 1998). The temperature and emissivity separation algorithm accurately retrieves surface temperature using the ASTER multispectral thermal infrared (TIR) bands (Gillespie et al., 1998). QuickBird images with resolutions of $2.8 \mathrm{~m}$ were used to make the detailed land cover classification. In addition, parcel (tax lot) level urban structural information and the building footprint were downloaded from the 
NYC Department of City Planning (NYC DCP, 2020). The urban structural parameters include building footprint area, building classes, land use category, commercial area, residential area, total floors, building heights, and year built. According to the year built, more than $95 \%$ of the buildings were built before 2000. Tree census data of 2005 in NYC census tracts were also used in the analysis (NYC DEP, 2020).

\subsection{Image classification}

Urban areas represent a wide range of land uses and surface properties. To fully understand the spatial characteristics of urban structural and anthropogenic factors and the feedback to humandominated urban ecosystems, we classified the urban areas into different surface types. The most common subdivisions of urban areas are residential (low, medium, and high), industrial, commercial areas (Loveland and Belward, 1997). Each subdivision has distinct surface morphological characteristics defined by the amounts and types of vegetation and the size or roughness of different elements. These differences result in variations of flux partitioning across a city and the development of distinct micro- to local-scale climates. However, we used a slightly different classification scheme for different urban components based on spectral reflectivity of multispectral (blue, green, red, and near-infrared wavelengths) QuickBird satellite.

We used a supervised approach to classify the very high-resolution QuickBird imagery into various urban land categories (bright, medium bright, and dark impervious surfaces, shadows, trees, grassland, and bareground. We used the Fuzzy ARTMAP for classification, a neural network developed by Carpenter et al. (1992). This algorithm has been widely used for satellite image classification (Carpenter et al., 1999a,b; Pax-Lenney et al., 2001). The ARTMAP is a match-based 
learning neural network that uses a self-organizing arbitrary system to map inputs to outputs. It also has attractive features such as being fast and stable (Carpenter et al., 1999a,b).

We selected training areas for shadows, bright, medium bright, dark impervious surfaces, trees, grassland, and bareground. The spatial resolution of the QuickBird image is fine enough to distinguish these individual features represented by urban materials such as pavement, rooftops, trees, or bareground. The shadow category includes shadows cast by buildings and trees; bright impervious surfaces correspond to bright surfaces seen in the original satellite images, including most rooftops and industrial plants. Medium-bright surfaces are mainly concrete materials, and dark surfaces are mainly asphalt and tar. Dark surfaces appear brighter compared to shadows. Trees and grasses are from parks and sides of streets, and bareground refers to playing fields.

\subsection{Accuracy assessment}

To quantitatively evaluate the accuracy of our image classification results, we used both confusion matrix and further visual interpretation. To create the confusion matrix, we recollected our ground truth from the original image through visual interpretation and our knowledge of New York City. The ground truth used to create a confusion matrix is wholly independent of the training sites for our classification algorithm. In reality, confusion matrix analysis is not a panacea for classification accuracy assessment (Friedl et al., 2000). We collected large homogeneous areas as ground truth and avoided mixed or heterogeneous areas. The accuracy assessed using the confusion matrix tends to be overestimated. This problem is even worse for the urban area due to the small scale of the urban structure, resulting in many mixed pixels even for high-resolution QuickBird images.

Most of the pixels are correctly classified (Table 1). However, some grasslands are misclassified as medium-bright impervious surfaces, while some dark impervious surfaces are 
misclassified as medium bright surfaces or the shadow category. A few tree pixels are classified as grassland, a few medium bright impervious surface pixels are classified as bright surfaces, and a small part of bright surfaces are misclassified as medium bright surfaces. Overall the classification accuracy is 0.959 , and the Kappa coefficient is 0.947 .

Table 1 . The confusion matrix shows the number of correctly and incorrectly classified pixels for the QuickBird classification map.

\begin{tabular}{|c|c|c|c|c|c|c|c|c|}
\hline Class & Shadow & $\begin{array}{l}\text { Bright } \\
\text { cover }\end{array}$ & $\begin{array}{l}\text { Impervious- } \\
\text { medium }\end{array}$ & $\begin{array}{l}\text { Impervious- } \\
\text { dark }\end{array}$ & Trees & Grassland & Bareground & Total \\
\hline Shadow & 5794 & 0 & 0 & 282 & 0 & 0 & 0 & 6076 \\
\hline $\begin{array}{l}\text { Bright } \\
\text { cover }\end{array}$ & 0 & 4045 & 14 & 0 & 0 & 0 & 0 & 4059 \\
\hline $\begin{array}{l}\text { Impervious- } \\
\text { medium }\end{array}$ & 0 & 49 & 2634 & 131 & 0 & 232 & 0 & 3046 \\
\hline $\begin{array}{l}\text { Impervious- } \\
\text { dark }\end{array}$ & 60 & 0 & 1 & 4468 & 0 & 0 & 0 & 4529 \\
\hline Trees & 0 & 0 & 0 & 0 & 725 & 0 & 0 & 725 \\
\hline Grassland & 0 & 0 & 0 & 0 & 13 & 473 & 0 & 486 \\
\hline Bareground & 0 & 2 & 0 & 0 & 0 & 0 & 398 & 400 \\
\hline Total & 5854 & 4096 & 2649 & 4881 & 738 & 705 & 398 & 19321 \\
\hline
\end{tabular}

Note: Overall accuracy is 0.959 , and the Kappa coefficient is 0.947 .

For the visual assessment of the shadow category, shapes turned out to be an effective indicator. The shapes of shaded areas projected on the ground should reflect the original shapes of the materials that obscured sunlight. More importantly, they should incline to the specific direction precisely opposite of the Sun's position. For instance, the dark rectangular area which does not align with the Sun's direction was almost automatically eliminated from the shadow category. The shadow cover includes the shadows cast by both trees and buildings. Our visual interpretation indicates that we classify the shadow category very well. 


\subsection{Data processing}

The data were cleaned, processed, merged, and analyzed in the python environment. We process the following data for analysis: 1) NYC census tract with demographic information, 2) Parcel level attributes containing building footprint, structural, and land use information, 3) Tree census data containing location information for NYC region, which is filtered for alive trees, 4) Land use classification of QuickBird image, and 5) ASTER surface temperature at $90 \mathrm{~m}$ spatial resolution.

We used zonal statistics functions in the python environment to aggregate ASTER surface temperature at the census tract level. The zonal statistics provide minimum, maximum, mean, and majority, including several other statistical parameters (Rasterstat, 2021). We aggregated total trees, building area, residential area, commercial area, garage area, and retail areas within each census tract to compare the relationships between surface temperatures and different building attributes. We computed fractions of different QuickBird land use categories within each ASTER pixel to establish a relation between surface temperature and the fraction of different urban components. The data provides information on the percentage of each QuickBird land cover class within each ASTER surface temperature pixel of $90 \mathrm{~m}$ resolution. We also computed the dominant land use category based on the building footprint record for every census tract. All the figures were prepared in R studio, ArcGIS Pro 2.8.1, and Python.

\section{Results}

\subsection{Satellite-derived surface temperature and urban compositions}

The ASTER data shows that surface temperature increases from the coast to inland, with cool surface temperatures in Staten Island and South Brooklyn to the warmest in New Jersey in the west 
(Fig. 2b). Three significant areas of temperature patterns could be identified, with the coolest coastal areas in Brooklyn, then the warmer Manhattan, Queens and the Bronx, and the warmest New Jersey areas. These surface temperature patterns correspond to two ridges (Fig. 2a), which block the sea breeze to bring cooler air inland.

A visual inspection of our supervised classification map of the NYC area (Manhattan and adjoining region) reveals that the distribution of different urban materials and landforms appears to be realistic (Fig. 3a). Water bodies are represented by the two rivers and lakes in Central Park, green patches of Central Park, tracts of large paved urban surfaces, and buildings that line the streets are all found at their expected locations. Further, the shadow is well characterized in the downtown Manhattan area, where most high-rise buildings with significant shadow components are located. The lack of bareground class also indicates the efficient use of space in the NYC area, where the land value is relatively high. The medium-dark and dark impervious surfaces represent the two dominant classes in our study area (Fig. 3b). Trees and shadows are the second most dominant classes, followed by water bodies, bright surfaces, and grassland.

Brighter surfaces have the highest mean surface temperature $(312.6 \mathrm{~K})$. While the lower mean surface temperature was represented by shadow $(310 \mathrm{~K})$, typically cast by high-rise buildings, grassland (308 K), trees (307.6 K), and water bodies (303.3 K) (Fig. 3c). Most of the shadow pixels are in Manhattan, whereas brighter pixels are in Queens. Some of those bright surfaces are industrial plants, which release waste heat. In Manhattan, the dark, heat-trapping materials, like tar roofs and asphalt roads blocked from direct exposure to incoming solar radiation, exhibit cooler surface temperatures (Fig. 3a, 2b). By contrast, areas to the west of midtown Manhattan and the north of lower Manhattan display much warmer surface temperatures (Fig. 3a, 2b). These areas are dominated by low-rise buildings that cast fewer shadows. Three airports in this region, 
including La Guardia in Queens, JFK in Brooklyn, and Newark in New Jersey, all show hotspots. These hotspots correspond to the area with large bright impervious surfaces, indicating that large impervious surfaces exhibit greater surface temperature, even if the surface is bright.
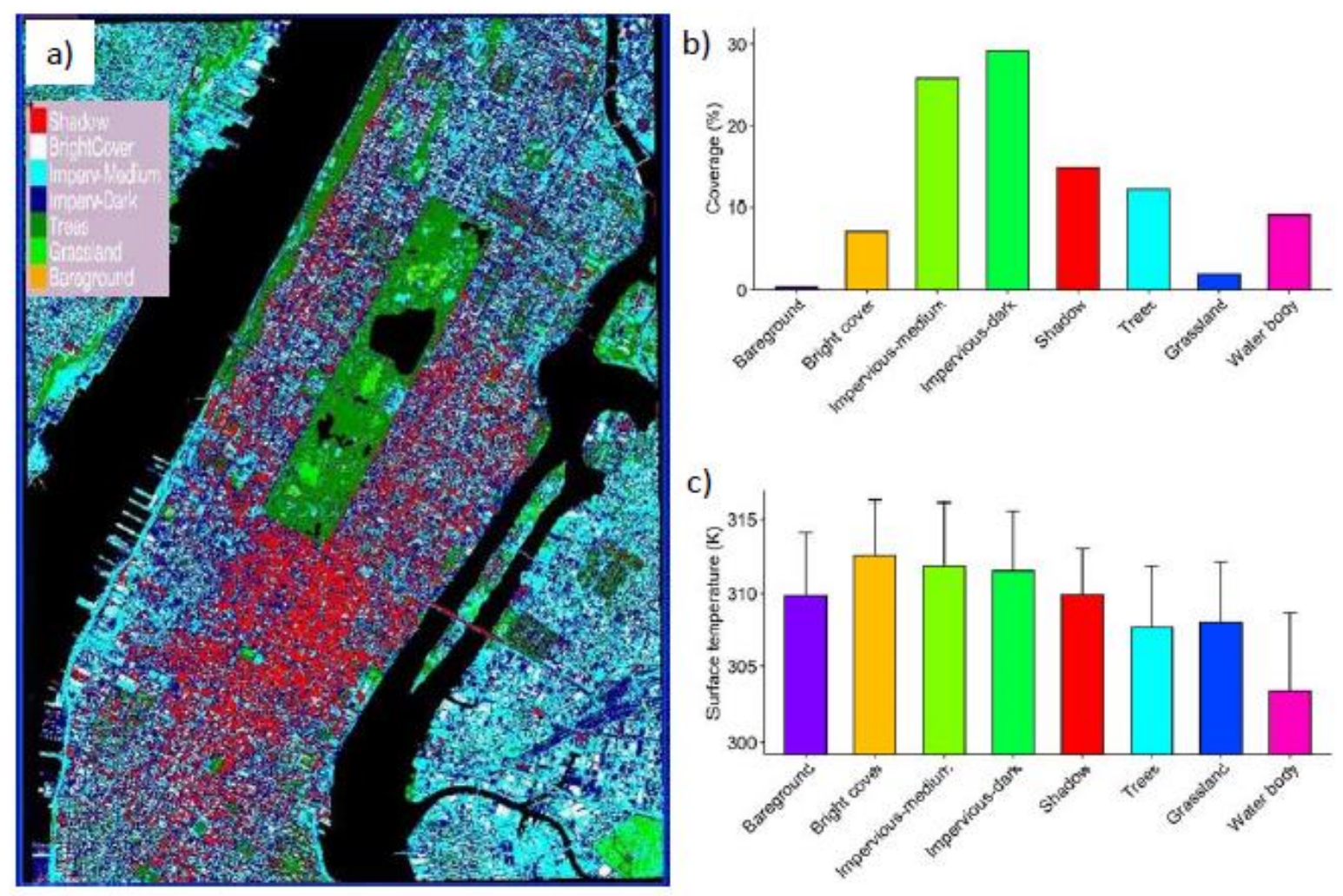

Figure 3. The figure shows: a) The composition of urban materials obtained by classification of the QuickBird image using Fuzzy ARTMAP neural network scheme, b) Total coverage of each urban component in Manhattan and its surrounding area, and c) Bar chart of surface temperature (mean and standard deviation) in different urban components.

\subsection{Relationship between urban compositions and surface temperature}

The urban land cover compositions derived from the QuickBird image were co-registered with the ASTER surface temperature. The fractional cover of QuickBird classes within each ASTER pixel was calculated. We assumed that a minimum of $10 \%$ pixel of each QuickBird class should substantially impact surface temperature in each ASTER pixel. The analysis revealed that surface temperature decreases with the increase in shadow coverage (Fig. 4a). The Sun position when the 
ASTER data was collected (52-degree Sun elevation angle) was lower than the Sun position when the QuickBird data was collected (63-degree Sun elevation angle), so the ASTER image has more shadows than the QuickBird image. As discussed below, this may not affect the relationship between surface temperature and shadows but may add noise to the relationships between surface temperature and impervious surface classes.

The surface temperature decreases with tree cover, with a steeper decrease than the shadow class (Fig. 4b). For the dominant grassland class, surface temperature shows no relationship (Fig. 4c). Grassland does not reduce and amplify urban heat, consistent with the ground observations conducted in a tropical city by Nichol and Wong (2005). Surfaces with brighter cover show an increasing surface temperature trend (Fig. 4d). Usually, bright surfaces with high albedo reflect more and absorb less solar radiation than other impervious surface materials. We expect that surface temperature decrease with bright surface cover. However, due to its low heat capacity, even the bright impervious surface can heat the surface easily and quickly. Also, the bright surface is surrounded by heat-trapping surfaces such as dark and medium-dark impervious surfaces. Those nearby darker materials may amplify and increase the surface temperature with bright covers. Another contributing factor could be that most bright surfaces are rooftops and industrial plants, constantly exposed to the Sun. Therefore, the presence of co-existing darker impervious materials within the same ASTER pixel of $90 \mathrm{~m}$ spatial resolution.

The relationship is quite noisy for the dominant medium-dark impervious surface class, and the overall surface temperature increases with the medium-dark surface cover. However, the slope is not very steep (Fig. 4e). The main reason for the noise is that the definition for this class is not very clear, and many medium-dark surfaces can have different materials. Those materials can have different radiative emission and absorption properties that affect the surface temperature 
dramatically. The surface temperature increases with the impervious dark surface cover (Fig. 4f).

The slope is slightly steeper than the medium-dark surface cover.
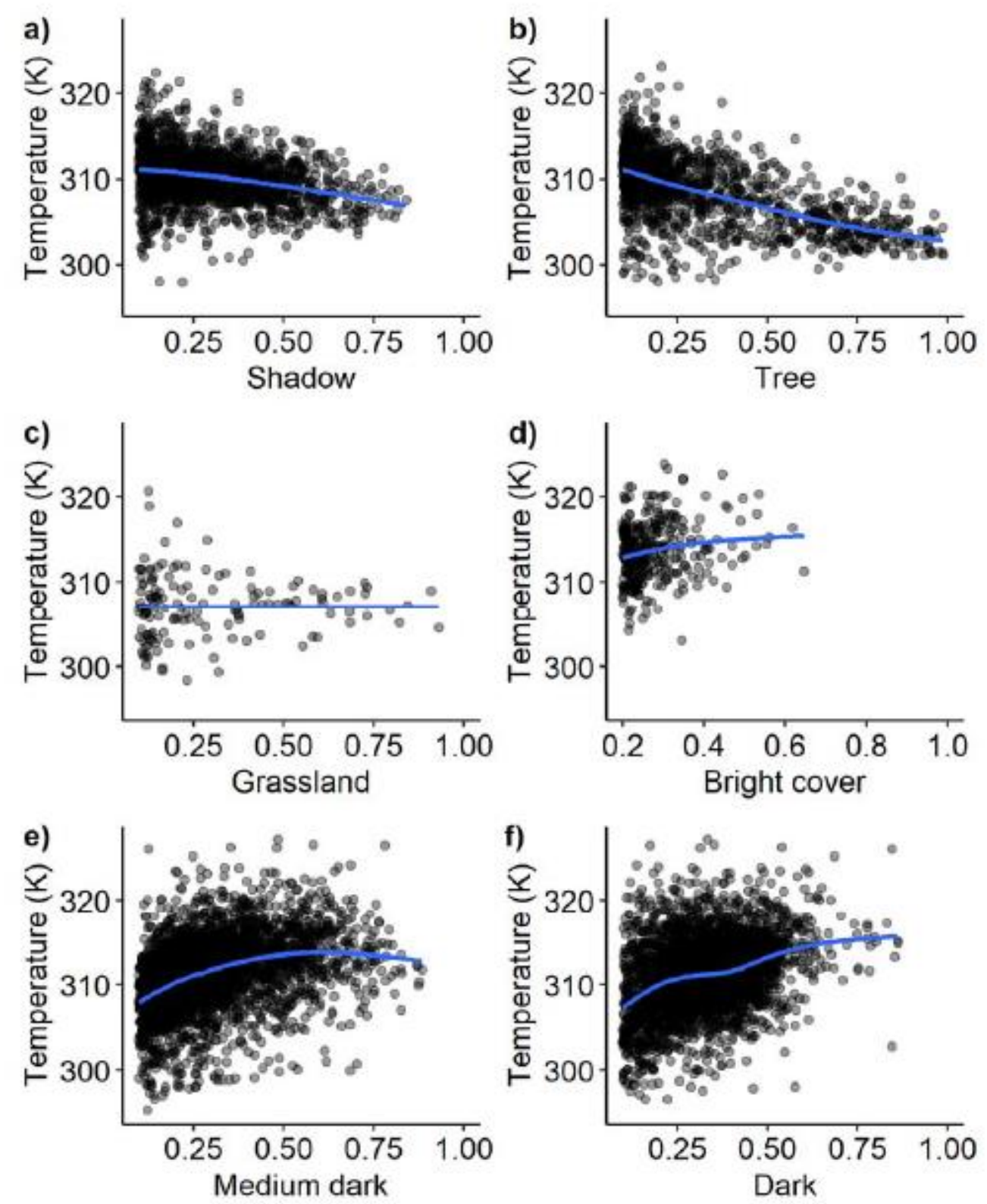

Figure 4. Relationship between ASTER surface temperature $(\mathrm{K})$ and the fraction of different urban components $>10 \%$. Solid lines show a locally weighted scatterplot smoothing (LOWESS) function. Sub-plot (a-f) shows individual relationships of urban components with surface temperature. 


\subsection{Impact of land use and building structure on surface temperature}

The mean surface temperature for building footprint in the Bronx is the highest, followed by Brooklyn, Manhattan, and Queens. Building footprint data indicate that the mean surface temperature in Staten Island is the lowest, at least $1.5 \mathrm{~K}$ less than that in Queens and $4 \mathrm{~K}$ less than that in Bronx and Brooklyn (Fig. 5). The analysis revealed that the industrial and manufacturing land use category generates significantly more surface heat than any other land use category (Fig. 6). In addition, transportation and utility, and parking facilities are also producing higher surface heat, amongst others. Public facilities and institutions, open space and outdoor recreations, and one and two-family buildings comprise land use categories producing the lowest surface heat. The choropleth map showed that the heat-generating industrial and manufacturing land use is dominant in southwestern Queens and the Bronx, where surface temperature is more than $313 \mathrm{~K}\left(40^{\circ} \mathrm{C}\right)$ and forms a high-temperature hotspot (Fig. 7). In parts of Bronx, Brooklyn, and Queens, multi-family walk-up buildings are also associated with higher surface temperature clusters. The possibility of anthropogenic factors such as winter heating and summer cooling is likely generating more heat in this locality. 


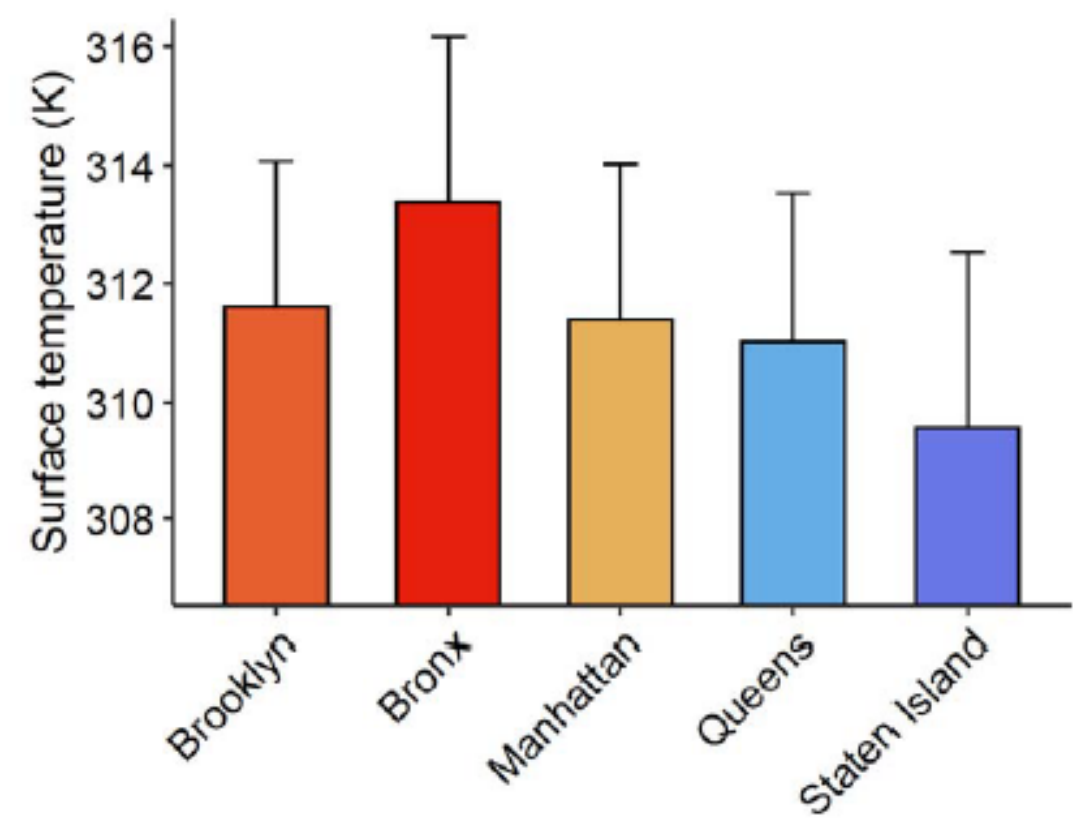

Figure 5. The bar chart shows surface temperature (mean and standard deviation) in five boroughs of New York City.

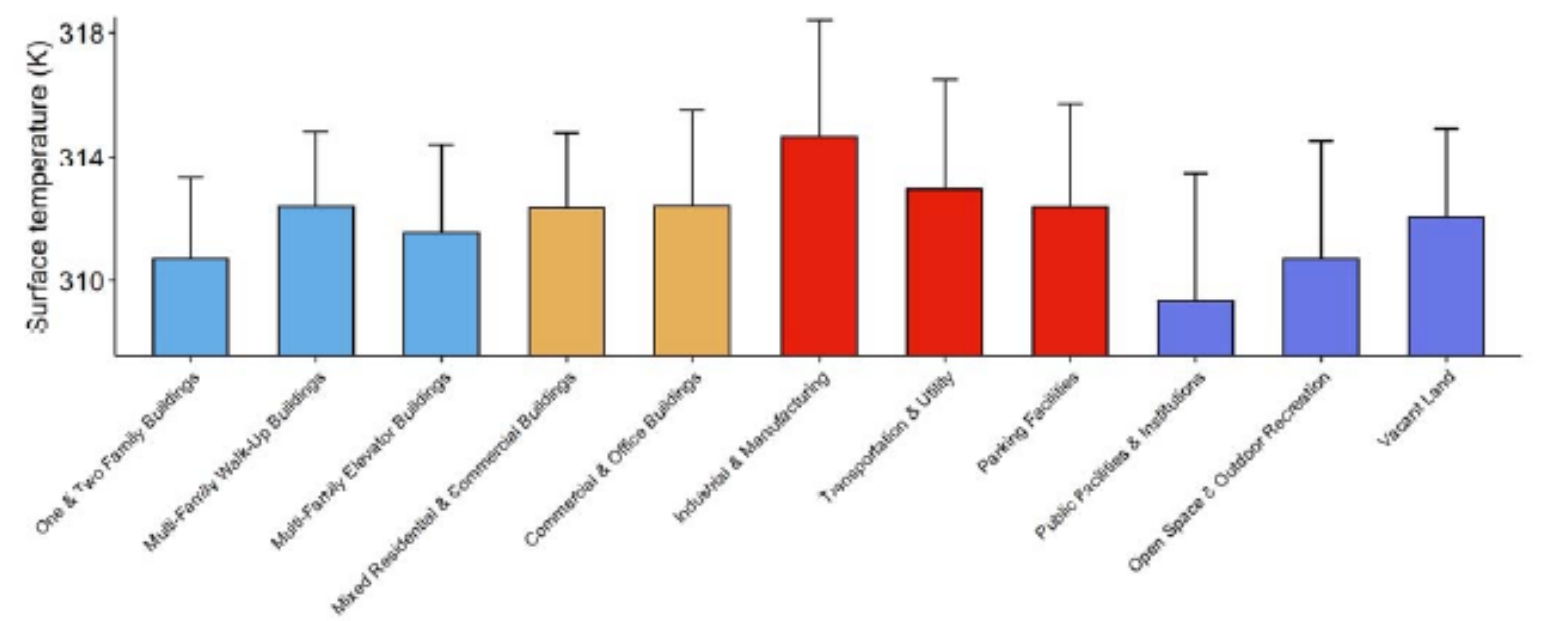

Figure 6. The bar chart shows surface temperature (mean and standard deviation) in individual building's land use category in New York City. The color was assigned to residential land use categories of similar characteristics. 


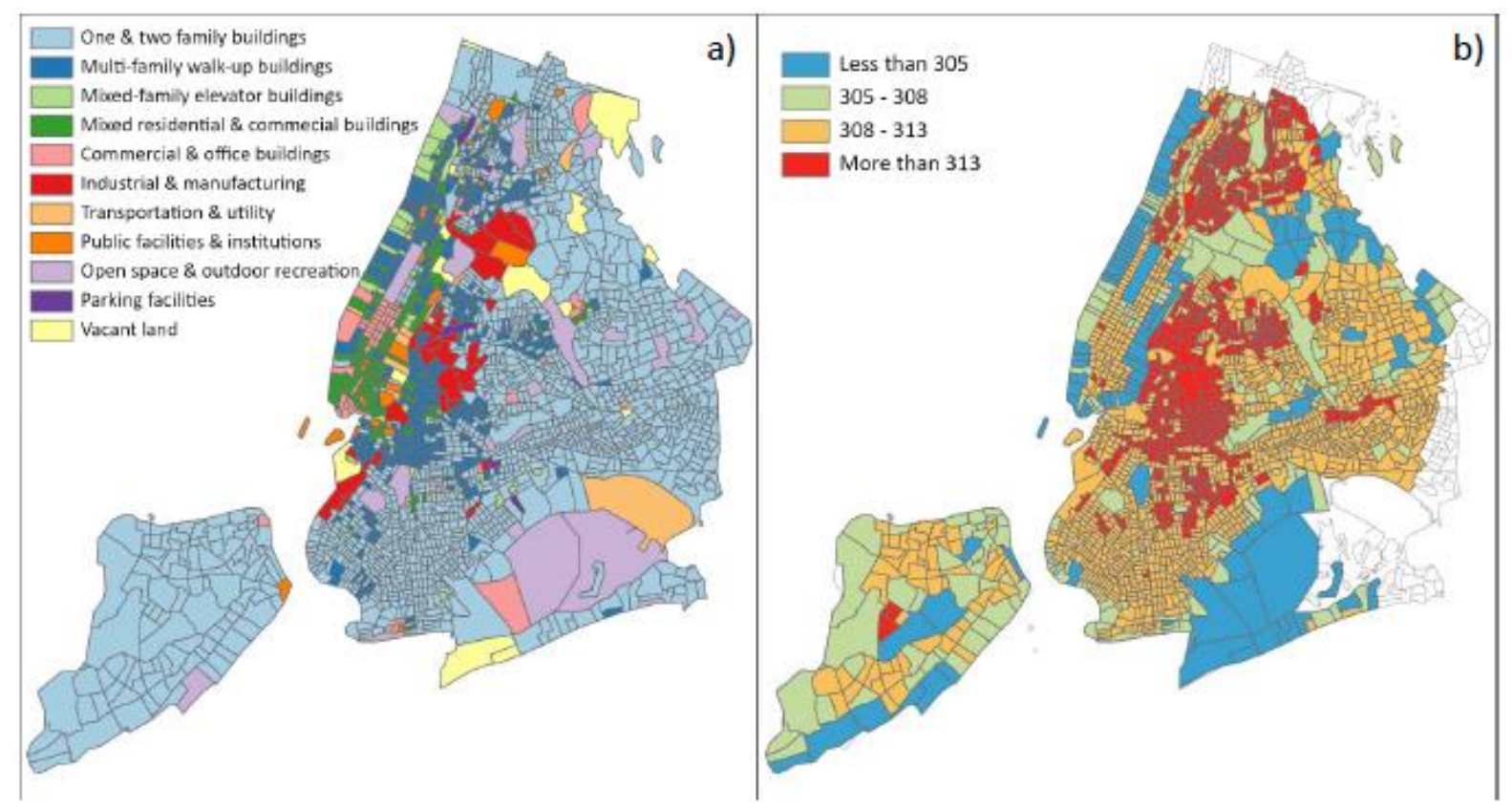

Figure 7. Choropleth maps show: a) Dominant building's land use and b) Surface temperature (mean, K) in different census tracts of New York City.

The relationships between surface temperature and the number of trees in different NYC census tracts show that higher surface temperature is associated with lower tree counts, although some exception exists (Fig. 8a). Most of these census tracts are located in Bronx, Brooklyn, and Queens. However, low tree counts are also associated with lower surface temperatures in some of the census tracts. Those census tracts are primarily located in Manhattan. The scatter plot of the relationship between surface temperature and building height reveals an interesting pattern (Fig. 8b). Higher surface temperature is associated with lower building heights (i.e., fewer floors), whereas lower surface temperature is associated with higher heights (i.e., more floors). In addition to that, we observed that the surface temperatures increase with building density but show decreasing trend when density is higher than 0.4 (Fig. 9a). The scatter plot also revealed that surface temperature decreases with higher building height variability (Fig. 9b). Most of these census tracts that show a decrease in surface temperature are in Manhattan, where buildings are 
much taller with more floors and height variability. We also show that census tracts with less building height variability and higher tree counts are associated with lower surface temperature. These results indicate that the larger variations in building heights and trees contribute to lower surface temperature in urban areas of New York City during autumn. The combination of the above factor increases shadows cast by higher-rise buildings on the surrounding lower-rise buildings causing cooling effects. The observed heterogeneity in surface temperature is likely associated with the neighborhood's specific building land use and structure. We observed that Manhattan has a greater share of high-rise buildings, while Staten Island has more trees than other localities. On the contrary, Bronx, Brooklyn, and Queens have a greater share of industrial and manufacturing land use and multi-family walk-up buildings, including fewer trees. 


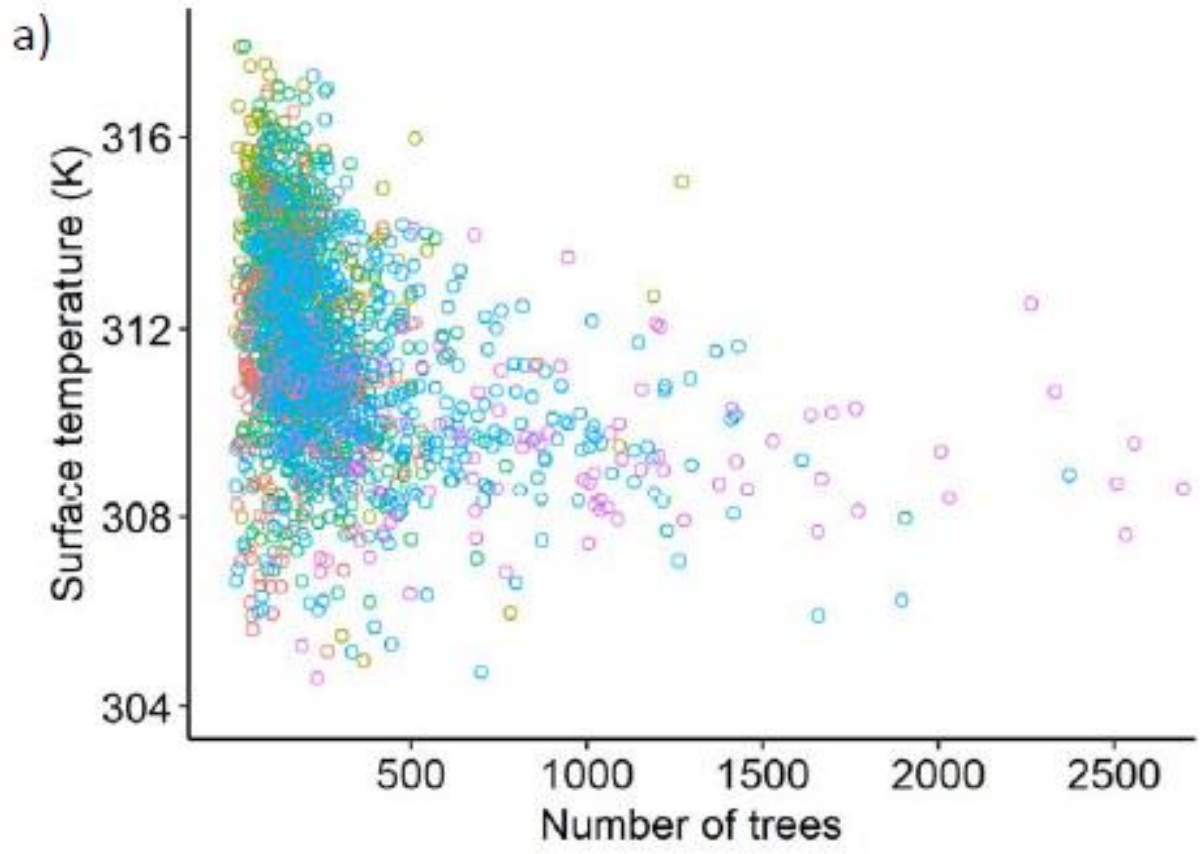

$M N \circ B X \circ B K \circ Q N \circ S I$

b)

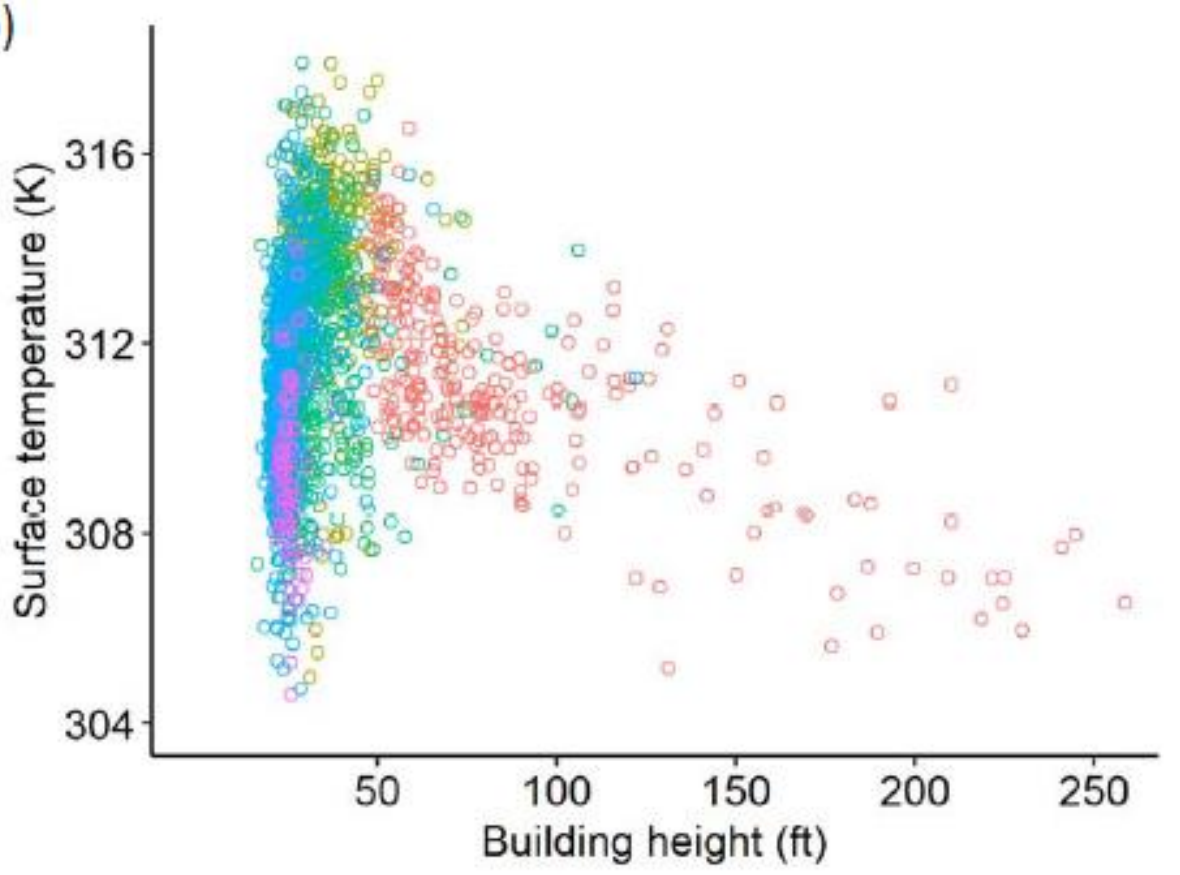

$M N \circ B X \circ B K \circ Q N \circ S I$

Figure 8. The scatterplot shows the relationship between: a) surface temperature (mean) and the number of trees, and b) surface temperature (mean) and building height in different census 
tracts of New York City. Different colors signify boroughs of New York City: MN for Manhattan, BX for Bronx, BK for Brooklyn, QN for Queens, and SI for Staten Island. 


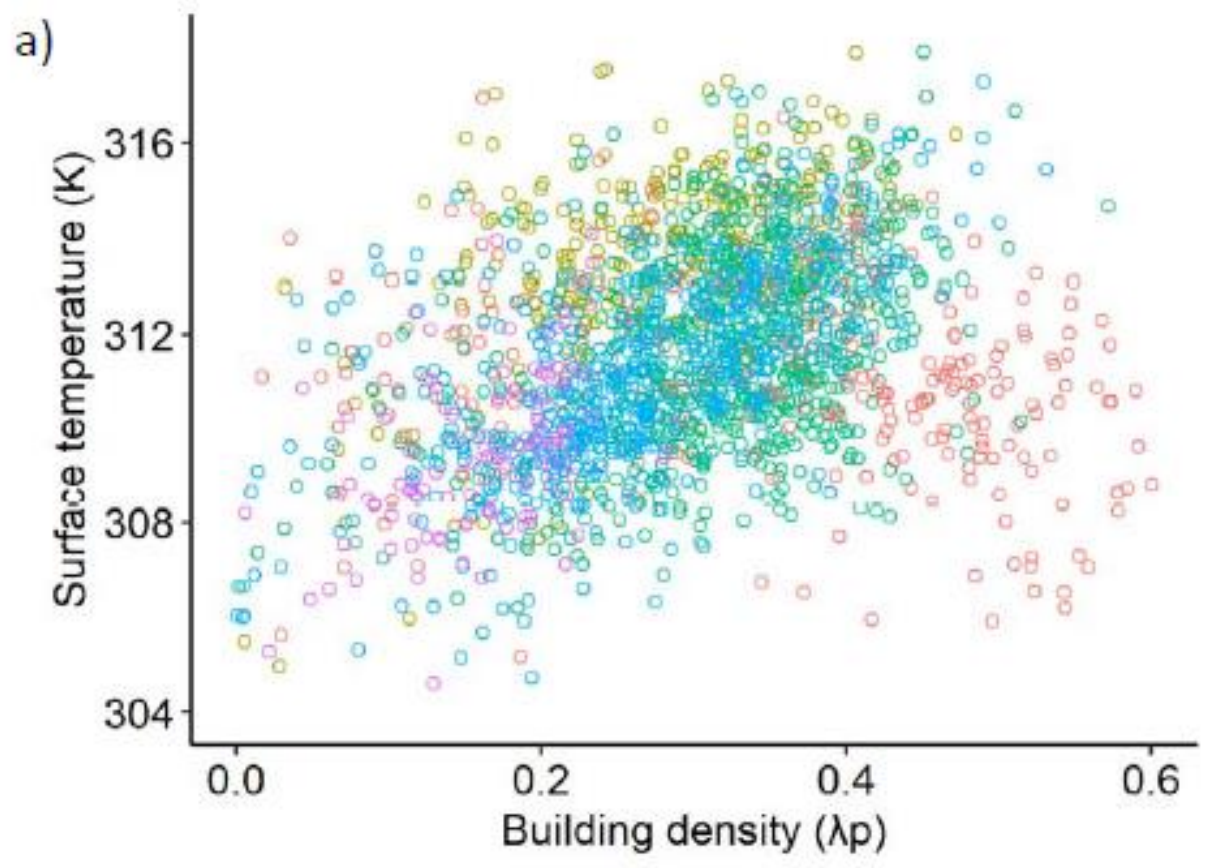

$M N \circ B X \circ B K \circ Q N \circ S I$

b)

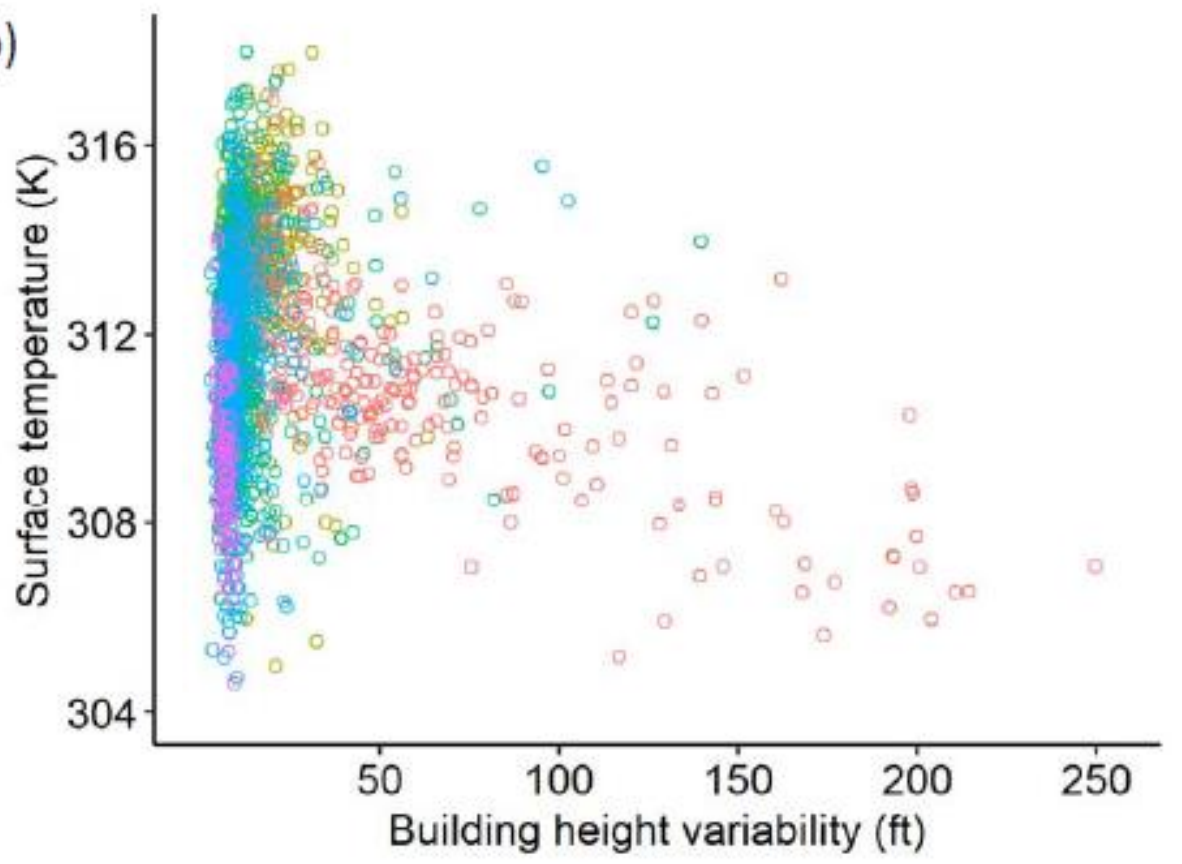

$M N \circ B X \odot B K \odot Q N \circ S I$

Figure 9. The figure shows the relationships between: a) Surface temperature (mean) and building density (ratio of the total building footprint to the land area), and b) surface temperature (mean) and building height variability in different census tracts of New York City. Different 
colors signify boroughs of New York City: BK - Brooklyn, BX - Bronx, MN - Manhattan, QN - Queens, and SI - Staten Island.

\section{Discussion}

Three factors, trees, building heights, and impervious surfaces, including bright surfaces, are primarily responsible for surface temperature heterogeneity in our study site. The replacement of vegetation by heat-trapping and non-porous urban materials alters surface conditions such as albedo, thermal capacity, and heat conductivity. Such transformation alters radiative fluxes between the surfaces and the lower atmosphere (Oke, 1982).

Trees reduce heat in two ways. Firstly, the shadows resulting from the tree control the total amount of radiation absorbed per unit surface area of heat-trapping materials. Secondly, trees return more surface heat to the atmosphere through evapotranspiration and reduce surface temperatures. Similarly, shadows cast by high-rise buildings reduce the amount of solar energy absorbed by the urban heat-trapping surfaces, and so there will be less heating effect. Guo et al. (2016) observed a positive effect of building height and building density on land surface temperature. They further observed higher land surface temperature associated with medium building height and a lower building density. Krüger et al. (2011) showed a direct link between urban climate and building heights. However, we show that surface temperature decreases with an increase in building heights variability. Generally, the shadows cast by tall buildings cover large urban impervious surfaces in the areas having more height variability. In addition, because the Sun is at a lower elevation in September cause longer shadows in comparison with the summer seasons. Likewise, Zheng et al. (2019) observed adverse effects of building heights on land surface temperature in residential areas of Beijing. 
The shadows from high-rise buildings influence temperature, similar to how vegetation affects surface temperature (Ni et al., 1999). Mutual shadows created by tall vegetation, such as in forests, eliminate any existing gaps in forests. Similarly, even if some gaps exist between high-rise buildings in cities like New York, the mutual shadows cast on the wall of the buildings and the ground will reduce heating effects. Additionally, when the height variability increases, the shadows can effectively cover the adjacent building walls up to hundreds of meters away depending on the Sun's azimuth (Vo and Laefer, 2019). Under such conditions, less incident radiation will likely be absorbed on the urban surfaces (horizontal and/or vertical), leading to cooler urban surfaces. Wang and Xu (2021) also indicate that land surface temperature decreases significantly with building height differences and brings a cooling effect.

Our results show that surface temperature increases with increasing fractions of impervious cover (both dark and medium-dark). This indicates that dark urban surfaces, mainly low-rise multifamily walk-up buildings with an average number of floors of 3.15, produce fewer shadows contribute to the urban heat. Surfaces with brighter cover show an increasing surface temperature trend. Usually, bright surfaces increase surface albedo, i.e., reflect more and absorb less solar radiation than other impervious surface materials (Deng et al., 2021). It is expected that surface temperature would decrease with bright surface cover due to its high albedo. However, due to its low heat capacity, even the bright impervious surface can heat the surface easily and quickly. Most bright surfaces are rooftops and industrial plants, which are constantly exposed to the Sun. Also, the bright surface is surrounded by dark and medium-dark heat-trapping surfaces. The presence of these heat-trapping darker materials may amplify the surface temperature. For the dark and medium-dark impervious surfaces and roofs, not much light penetrates the surfaces, incident radiation is not reflected back to the atmosphere is used to heat the surfaces, causing increased 
surface temperature. Using high spatial resolution satellite data, we characterized the shadows, green vegetation, impervious surfaces, and their brightness and identified each component's impact on surface temperature in our study site.

Using building footprint data at the parcel level combined with high-resolution satellite data we identified the effects of different urban components such as shadows, green vegetation, building heights, building density, impervious surfaces, and their brightness on surface temperature. Building footprint data reinforces the satellite-derived interpretation of finer-scale surface temperature variations in urbanized settings and determines the likely causes. Our analysis indicates that shadows cast by high-rise buildings containing multiple floors and trees reduce UHI effects. In contrast, dark and medium-dark impervious surfaces, typically low-rise multi-family walk-up buildings, and brighter industrial and manufacturing areas increase UHI.

Various environmental and governmental agencies are working towards mitigating the UHI effect. Two approaches are being adopted. One uses highly reflective materials for roofs and pavements, and the other planting trees. The green roof programs are being conducted and tested in several big cities, including New York City (Treglia et al., 2018). Green roofs impact stormwater use and help release heat absorbed on roof surfaces and cool overall surface temperature. Our study indicates that trees and grasses can mitigate UHI. However, most green roof projects are implemented in Manhattan, where ASTER data show much cooler surface temperatures during autumn than surrounding areas (Fig. 10). Therefore, the green roofs program can be implemented and tested in the areas producing more heat during autumn, especially in the areas dominated by multi-family walk-up buildings and industrial and manufacturing land use. In addition, New York City adopted cool roof projects by whitening the black asphalt rooftops and make them highly reflective to reduce surface temperature (NYC CoolRoofs, 2021). However, it is essential to have 
a detailed analysis of the seasonal variations in surface temperature patterns and to test the longterm effect of cool roofs on urban heat in the presence of other impervious surfaces.

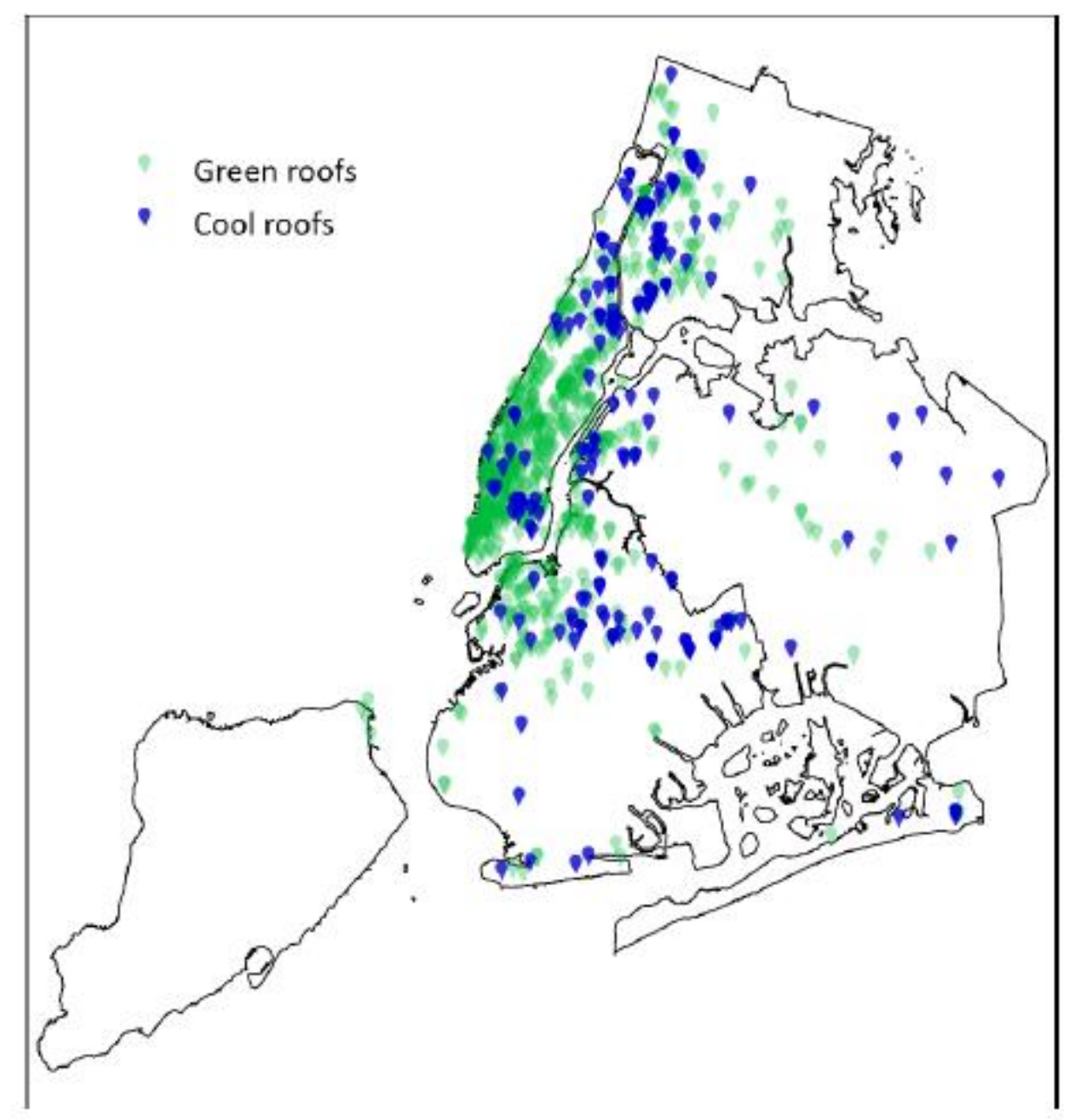

Figure 10. Maps showing building locations with cool roofs in 2012 and green roofs in 2016 in New York City.

The future work will involve simulating time-series surface temperature patterns as a function of urban structures, vegetation cover, and shadows derived from high-resolution satellite data for the entire NYC region. It is well known that building shadows show strong seasonality and affect urban land surface temperature (Yu et al., 2019). Therefore, we planned to use high-resolution ECOSTRESS images to study the spatiotemporal pattern of urban heat. In particular, we will 
model the shadow effects due to building height variation and trees on surface temperature patterns. We will combine the locations of cool roofs and green roofs and quantify the cumulative effect on reducing the surface temperature in the city to help design priority locations since hightemperature hotspots during autumn are in the south Bronx, southeastern Queens, and northern Brooklyn. Our long-term goal is to assimilate satellite-observed spatiotemporal patterns of urban heat into urban climate models. The shadow analysis would provide helpful information in sustainable urban design, including access to sunlight during different seasons, and help mitigate the impact of climate change. The quantification of UHI effects has a potential public health implication and help safeguard people from heat-related stress.

\section{Conclusion}

Our study shows that the trees and shadows cast by high-rise buildings and their variability have a cooling effect. In contrast, more impervious surfaces show a heating effect even in the presence of highly reflective bright surfaces. The census tract with industrial and manufacturing areas and multi-family walk-up buildings as dominant land use categories correspond to the highest surface temperature. Buildings with lower heights (fewer floors) and height variability are associated with higher surface temperature. Although the building density is the highest in Manhattan (the central business district), many tall buildings with variable heights have shown cooling effects. Staten Island has the lowest mean surface temperature amongst all boroughs of New York City, where the number of trees is more. While, Bronx has the highest mean surface temperature and constitutes moderate building density, height, and height variability. The finding from this study has an important implication for urban heat island modeling, especially the positive effects of trees and 
building shadows. It could help prioritize the areas to mitigate the UHI effect and reduce associated environmental and health-related costs, including sustainable urban planning.

\section{Author contributions}

Conceptualization and Methodology, B.N. and W.N.-M.; Software, B.N. and M.Ö.; Formal Analysis and Data Curation, B.N.; Writing - Original Draft Preparation, B.N.; Writing - Review \& Editing, B.N., M.Ö. and W.N.-M.; Funding Acquisition, W.N.-M. All authors have approved the content of the submitted and/or published version of the manuscript.

\section{Funding}

This study is funded by NASA grant number 80NSSC20K1718.

\section{Data availability statement}

ASTER Level 2 Surface Temperature image was downloaded from https://pdaac.usgs.gov/products/ast_08v003/ (Accessed 2021-03-28). Building footprint and extensive land use and geographic data at the tax lot level were downloaded from https://www1.nyc.gov/site/planning/data-maps/open-data/dwn-pluto-mappluto.page (Accessed 2021-04-03). Street tree census data were downloaded from https://data.cityofnewyork.us/Environment/2005-Street-Tree-Census/29bw-z7pj (Accessed 202104-05). New York City Cool Roofs location was downloaded from https://maps.princeton.edu/catalog/sde-columbia-cul_nyc_dob_coolroofs_2012. (Accessed on August 9, 2021). Green roof locations were downloaded from http://doi.org/10.5281/zenodo.1469674 (Accessed on August 9, 2021). 


\section{Conflicts of interest}

The authors declare no conflict of interest.

\section{Acknowledgment}

The authors would like to thank anonymous reviewers for their time and effort in reviewing this paper and improve its quality. We also thank Atsushi Tomita for his help in LULC classification and accuracy assessment.

\section{References}

ASTER_08, 2020. NASA/METI/AIST/Japan Spacesystems and U.S./Japan ASTER Science Team. ASTER Level 2 Surface Temperature Product. 2001, distributed by NASA EOSDIS Land Processes DAAC, https://doi.org/10.5067/ASTER/AST_08.003. (Accessed 2021-0705).

Callaghan, A., McCombe, G., Harrold, A., McMeel, C., Mills, G., Moore-Cherry, N., Cullen, W., The impact of green spaces on mental health in urban settings: a scoping review. J. Ment. Health, 30, 179-193, 2021.

Carlson, T. N., 1986: Regional-scale estimates of surface moisture availability and thermal inertia using remote thermal measurements. Remote Sens. Rev., 1, 197-247.

Carpenter G. A., Grossberg S., Markuzon N., Reynolds J. H., \& Rosen D. B. (1992). Fuzzy ARTMAP: a neural network architecture for incremental supervised learning of analog multidimensional maps. IEEE Transactions on Neural Networks, 3, 698-713. 
Carpenter, G.; Gopal, A.S.; Macomber, S.; Martens, S.; Woodcock, C.E. A neural network method for mixture estimation for vegetation mapping. Remote Sens. Environ. 1999, 70, 138-152.

Carpenter, G. A., S. Gopal, S. Macomber, S. Martens, and C. E. Woodcock. A neural network method for efficient vegetation mapping. Remote Sens. Environ. 70:326-338, 1999b.

Deng, Y.; Chen, R.; Xie, Y.; Xu, J.; Yang, J.; Liao, W. Exploring the Impacts and Temporal Variations of Different Building Roof Types on Surface Urban Heat Island. Remote Sens. 2021, 13, 2840. https://doi.org/10.3390/rs13142840

Friedl, M. A., C. E. Woodcock, S. Gopal, D. Muchoney, A. H. Strahler, C. Barker-Schaaf, A Note on procedures used for accuracy assessment in land cover maps derived from AVHRR data, International Journal of Remote Sensing, 21(5):1073-1077, 2000.

Gedzelman, S. D., S. Austin, R. Cermak, N. Stefano, S. Partridge, S. Quesenberry, and D. A. Robinson, Mesoscale aspects of the Urban Heat Island around New York City, Theor. Appl. Climatol., 75, 29-42, 2003.

Gillespie, A. R., Matsunaga, T., Rokugawa, S., and Hook, S. J., Temperature and Emissivity Separation from Advanced Spaceborne Thermal Emission and Reflection Radiometer (ASTER) Images. IEEE Transactions on Geoscience and Remote Sensing, 36, 1113-1126, 1998.

Guo G, Zhou X, Wu Z, Xiao R, Chen Y (2016) Characterizing the impact of urban morphology heterogeneity on land surface temperature in Guangzhou, China. Environ Model Softw $84: 427-439$.

Krüger EL, Minella FO, Rasia F (2011) Impact of urban geometry on outdoor thermal comfort and air quality from field measurements in Curitiba, Brazil. Build Environ 46:621-634. 
Loughner, C.P., Allen, D.J., Zhang, D-L., Pickering, K.E., Dickerson, R.R., Landry, L. Role of urban tree canopy and buildings in urban heat island effects: parameterization and preliminary results. J. Appl. Meteor. 51, 1775-1793, 2012.

Loveland, T. and Belward, A., 1997, The IGBP-DIS global 1km land cover data set, DISCover: first results, International Journal of Remote Sensing, 18,3289-3295. 1997.

Ni, W., Li X., Woodcock, C.E., Caetano, M.R. and Strahler, A. H., An Analytical Hybrid GORT Bidirectional Reflectance Model for Discontinuous Plant Canopies, IEEE Transactions on Geoscience and Remote Sensing, 37(2):987-999, 1999.

New York City Department of City Planning (NYC DCP), 2020. PLUTO: Extensive land use and geographic data at the tax lot level. https://www1.nyc.gov/site/planning/data-maps/opendata/dwn-pluto-mappluto.page

New York City Department of Environmental Protection (NYC DEP), 2020. 2005 street tree census, NYC Open data. https://data.cityofnewyork.us/Environment/2005-Street-TreeCensus/29bw-z7pj

New York City Cool Roofs (NYC CoolRoofs), 2012. Research Data Services (RDS), Columbia University Libraries and New York City Department of Buildings. https://maps.princeton.edu/catalog/sde-columbia-cul_nyc_dob_coolroofs_2012. (Accessed on August 9, 2021).

Nichol, J., Wong, M.S., 2005. Modeling urban environmental quality in a tropical city Landsc. Urban Plan. 73, 49-58.

Oke, T. R., The energetic basis of the urban heat island, Quart. J. Roy. Meteor. Soc. 108, 1-24, 1982. 
Pax-Lenney, M., C. E. Woodcock, S. A. Macomber, S. Gopal, and C. Song, Forest mapping with a generalized classifier and Landsat TM data, Remote Sensing of Environment, 77, 241-250, 2001.

Price, J. C., 1979: Assessment of the urban heat island effect through the use of satellite data. Mon. Wea. Rev., 107, 1554-1557.

Rasterstat, 2021. Zonal statistics: a method of summarizing and aggregating the raster values intersecting a vector geometry. https://pythonhosted.org/rasterstats/index.html

Roth, M., T. R. Oke, and W. J. Emery, 1989, Satellite-derived urban heat islands from three coastal cities and the utilization of such data in urban climatology, International Journal of Remote Sensing, 10:1699-1720.

Tan, H.; Ray, P.; Tewari, M.; Brownlee, J.; Ravindran, A. Response of Near-Surface Meteorological Conditions to Advection under Impact of the Green Roof. Atmosphere 2019, 10, 759. https://doi.org/10.3390/atmos10120759

Treglia, Michael L., McPhearson, Timon, Sanderson, Eric W., Yetman, Greg, \& Maxwell, Emily Nobel. (2018). Green Roofs Footprints for New York City, Assembled from Available Data and Remote Sensing (Version 1.0.0) [https://github.com/tnc-nyscience/NYC_GreenRoofMapping/blob/master/greenroof_gisdata/CurrentDatasets/GreenRo ofData2016_20180917.csv]. Zenodo. http://doi.org/10.5281/zenodo.1469674

Vo, A.V.; Laefer, D.F. A bid data approach for comprehensive urban shadow analysis from airborne laser scanning point clouds._ISPRS Annals of the Photogrammetry, Remote Sensing and Spatial Information Sciences, Volume IV-4/W8, 2019.

Voogt, J. A. and C. S. B. Grimmond, 2000. Modeling surface sensible heat flux using surface radiative temperatures in a simple urban area. Journal of Applied Meteorology, 39:1679-1699. 
Voogt, J. A. and Oke, T. R., 1997. Complete urban surface temperatures. Journal of Applied Meteorology, 36:1117-1132.

Voogt, J. A. and Oke, T. R., Thermal remote sensing of urban climates. Remote Sensing of Environment, 86:370-384, 2003.

Wang M, Xu H (2021) The impact of building height on urban thermal environment in summer: A case study of Chinese megacities. PLoS ONE 16(4): e0247786. https://doi.org/ 10.1371/journal.pone.0247786

Yamaguchi, Y.; Kahle, A.; Tsu, H.; Kawakami, T.; Pniel, M. Overview of Advanced Spaceborne Thermal Emission and Reflection Radiometer (ASTER). IEEE Trans. Geosci. Remote Sens. 1998, 36, 1062-1071.

Yoshikado, H. Vertical structure of the sea breeze penetrating through a large urban complex, J. Appl. Meteor., 29: 878-891, 1990.

Yoshikado, H., Numerical study of the daytime urban effect and its interaction with the sea breeze, J. Appl. Meteor., 31: 1146-1164, 1992.

Yu, K.; Chen, Y.; Wang, D.; Chen, Z.; Gong, A.; Li, J. Study of the Seasonal Effect of Building Shadows on Urban Land Surface Temperatures Based on Remote Sensing Data. Remote Sens. 2019, 11, 497. https://doi.org/10.3390/rs11050497

Zheng, Z.; Zhou, W.; Yan, J.; Qian, Y.; Wang, J.; Li, W. The higher, the cooler? Effects of building height on land surface temperatures in residential areas of Beijing. Phys. Chem. Earth 2019, $110,149-156$.

Zhou, B., Rybski, D. and Kropp, J. P., The role of city size and urban form in the surface urban heat island. Scientific Reports, 7, 4791, 2017. https://doi.org/10.1038/s41598-017-04242-2 
Zhou, D.; Xiao, J.; Bonafoni, S.; Berger, C.; Deilami, K.; Zhou, Y.; Frolking, S.; Yao, R.; Qiao, Z.; Sobrino, J.A. Satellite Remote Sensing of Surface Urban Heat Islands: Progress, Challenges, and Perspectives. Remote Sens. 2019, 11, 48. https://doi.org/10.3390/rs11010048 
